\title{
Full Stokes $I Q U V$ spectropolarimetry of AGB and post-AGB stars: probing surface magnetism and atmospheric dynamics
}

\author{
Agnès Lèbre $^{1 *}$, Michel Aurière ${ }^{2}$, Nicolas Fabas ${ }^{3}$, Denis Gillet ${ }^{4}$, \\ Eric Josselin ${ }^{1}$, Philippe Mathias ${ }^{2}$ and Pascal Petit ${ }^{2}$ \\ ${ }^{1}$ Laboratoire Univers et Particules de Montpellier, Université de Montpellier, \\ 34090 Montpellier Cedex 05, France \\ *email: Agnes.Lebre@univ-montp2.fr \\ ${ }^{2}$ Institut de Recherche en Astrophysique et Planétologie, Université de Toulouse, \\ 31400 Toulouse, France \\ ${ }^{3}$ Kiepenheuer-Institut fur Sonnenphysik \\ 79104 Freiburg, Germany \\ ${ }^{4}$ Observatoire de Haute Provence \& Observatoire Pytheas \\ 04870 Saint-Michel l'Observatoire, France
}

\begin{abstract}
Full Stokes spectropolarimetric observations of a Mira star ( $\chi$ Cyg) and a RV Tauri star (R Sct) are presented and analyzed comparatively. From their Stokes $V$ data (circular polarization), we report the detection of a weak magnetic field at the surface of these cool and evolved radially pulsating stars. For both stars, we analyse this detection in the framework of their complex atmospheric dynamics, with the possibility that shock waves may imprint an efficient compressive effect on the surface magnetic field. We also report strong Stokes $U$ and Stokes $Q$ signatures associated to metallic lines (as a global trend), those linear polarimetric features appear to be time variable along the pulsating phase. More surprising, in the Stokes $U$ and Stokes $Q$ data, we also detect signatures associated to individual metallic lines (such as $\mathrm{Sr}$ I $460.7 \mathrm{~nm}, \mathrm{Na} \mathrm{D}_{2} 588.9 \mathrm{~nm}$ ), that are known (from the solar case) to be easily polarizable in case of a global asymmetry at the photospheric level.
\end{abstract}

Keywords. stars: AGB and post-AGB, stars: atmospheres, stars: magnetic fields

\section{Introduction}

Among the cool and evolved stars, Mira and RV Tauri stars are radially pulsating targets. The Mira stars are located at the tip of the Asymptotic Giant Branch (AGB) i.e., the evolutionary stage of a low and intermediate mass star before its transition toward the post-AGB and Planetary Nebulae (PN) stages (Willson 2000), while the RV Tauri stars are in the post-AGB phase (Van Winckel et al. 1999). These variable stars share specific particularities such as photospheric convection, a complex atmospheric dynamics (including shock wave propagation), the presence of molecules, grains and dust in their upper (very extended) atmosphere and in their circumstellar envelope (hereafter CSE), and a prodigious mass loss (estimated up to $10^{-4} \mathrm{Msun} / \mathrm{yr}$ ). To date, the physical processes contributing to their high mass loss rates are not fully identified. Radiation pressure on dust and scattering effects of large grains are commonly invoked to drive mass loss. However, many unknowns remain, concerning in particular the generation of pulsations and their related atmospheric shock waves, the condensation and the opacity of dust grains in the upper atmosphere, the shaping of CSE, and the driving of its 


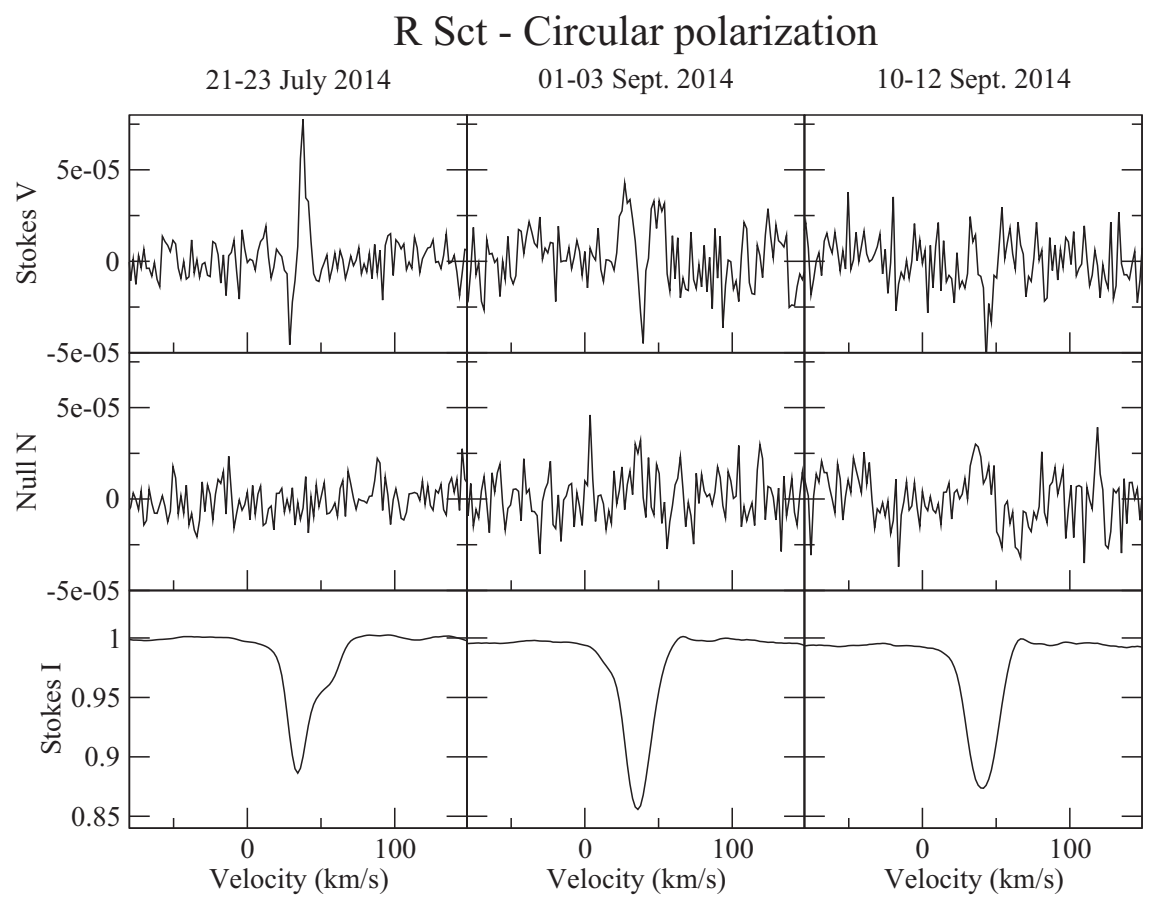

Figure 1. Circular polarization LSD profiles of the RV Tauri star R Sct (Stokes $I$, Null and Stokes $V$ profiles, from bottom to top panels); Narval observations collected in summer 2014 at 3 different epochs along the pulsation cycle (Period $=142$ days). Note that each of these observations is a combination of six Stokes $V$ sequences collected over 2 to 3 consecutive nights.

morphological evolution. Magnetic field is often invoked, beside binarity, to explain the shaping of the CSE during the post-AGB stage and the emergence of bipolar structures in the PN formation. Magnetic fields are thus potential agents in the mass loss process itself, as suggested by Pascoli \& Lahoche (2008) for Mira stars, and several recent works - based on radio-astronomy facilities - have indeed brought observational evidences for magnetic fields in the environment (i.e., CSE) of PN and of their AGB and post-AGB progenitors (see the review by Vlemmings 2011).

In order to investigate the magnetic field at the surface of variable cool and evolved stars (here, Mira and RV Tauri stars), we have carried out full Stokes IQUV spectropolarimetric observations with the Narval instrument mounted at Télescope Bernard Lyot (TBL) at Pic du Midi Observatory, France. Hereafter we present and discuss the observations of the Mira star $\chi$ Cyg and of the RV Tauri star R Sct, investigating their surface magnetic field (from circular polarization data - Stokes $V$ ) and atmospheric dynamics (from linear polarization data - Stokes $U$ \& Stokes $Q$ ). Both objects have been preferentially observed near or at a maximum light, i.e., when their atmospheric conditions were known to be the most favorable to limit the impact of heavy molecular absorption on the spectropolarimetric signals analyzed through the LSD software (Donati et al. 1997).

\section{Surface Magnetic Fields in cool and evolved stars}

In recent numerical MHD simulations, Thirumalai \& Heyl (2013) have proposed that a magnetic field would influence dust and wind formation in the prototype Oxygen-rich Mira (omi Cet), for which they have also predicted a surface field of a few gauss. As it 


\section{R Sct - Linear polarization}

15 July 2014

01 September 2014

11 September 2014

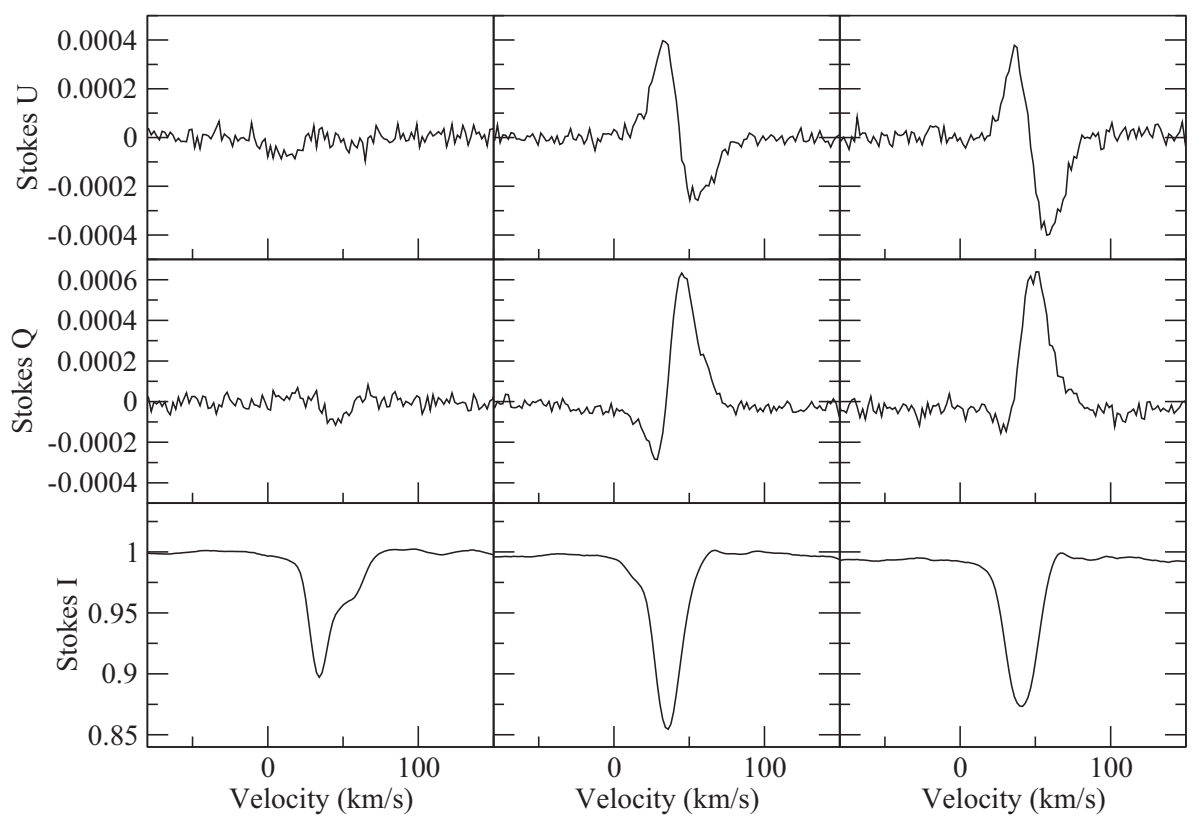

Figure 2. Linear polarization LSD profiles of the RV Tauri star R Sct (Stokes $I$, Stokes $Q$ and Stokes $U$ profiles, from bottom to top panels); Narval observations collected in summer 2014 at three different dates along the pulsation cycle (here and contrary to Figure 1, only one Stokes $U$ and one Stokes $Q$ sequences per night have been collected).

has been established for the cool red supergiant Betelgeuse (Dorch 2004), and because of their slow rotation (i.e., high Rossby number), convection in these cool and evolved stars cannot generate global magnetic fields, while local fields remain however possible and may be related to the generation of (at least) local, episodic mass loss events. Beyond this effect on mass-loss rate, magnetic fields are expected to shape the wind and to rule its efficiency to extract angular momentum from the star (as for cool main sequence stars). However, so far, little is known concerning magnetic fields at the surface of cool and evolved stars, especially in the case of the pulsating targets, Mira and RV Tauri stars. While in the past decade magnetic field have been investigated throughout their CSE (see for example the radiopolarimetric work of Herpin et al. 2006, dedicated to a large sample of Mira stars, including $\chi \mathrm{Cyg}$ ), surface magnetic fields have only been recently investigated and finally detected. From spectropolarimetric observations with Narval at TBL (ensuring a threshold detection below the gauss level), Lèbre et al. (2014) have detected a weak magnetic field at the surface of $\chi$ Cyg (surface field estimation: 2-3 G). Sabin et al. (2015) have also reported the presence of weak magnetic fields at the surface of two RV Tauri stars, U Mon and R Sct (surface field estimation: $10 \mathrm{G}$ and $1 \mathrm{G}$, respectively), among a sample of post-AGB stars. Both works have also revealed the existence of a strong connection between the surface stellar magnetism and the photospheric and atmospheric dynamics. Indeed, in the cases of $\chi$ Cyg and $\mathrm{R}$ Sct, the radiative shock wave known to propagate periodically throughout their stellar atmospheres (and to be responsible for peculiar spectroscopic features observed in visible and near infra-red spectra) seems to have an important connection with the surface magnetism and is supposed to act through compressive amplification on a weak surface field. For $\chi$ Cyg, this behavior is discussed 


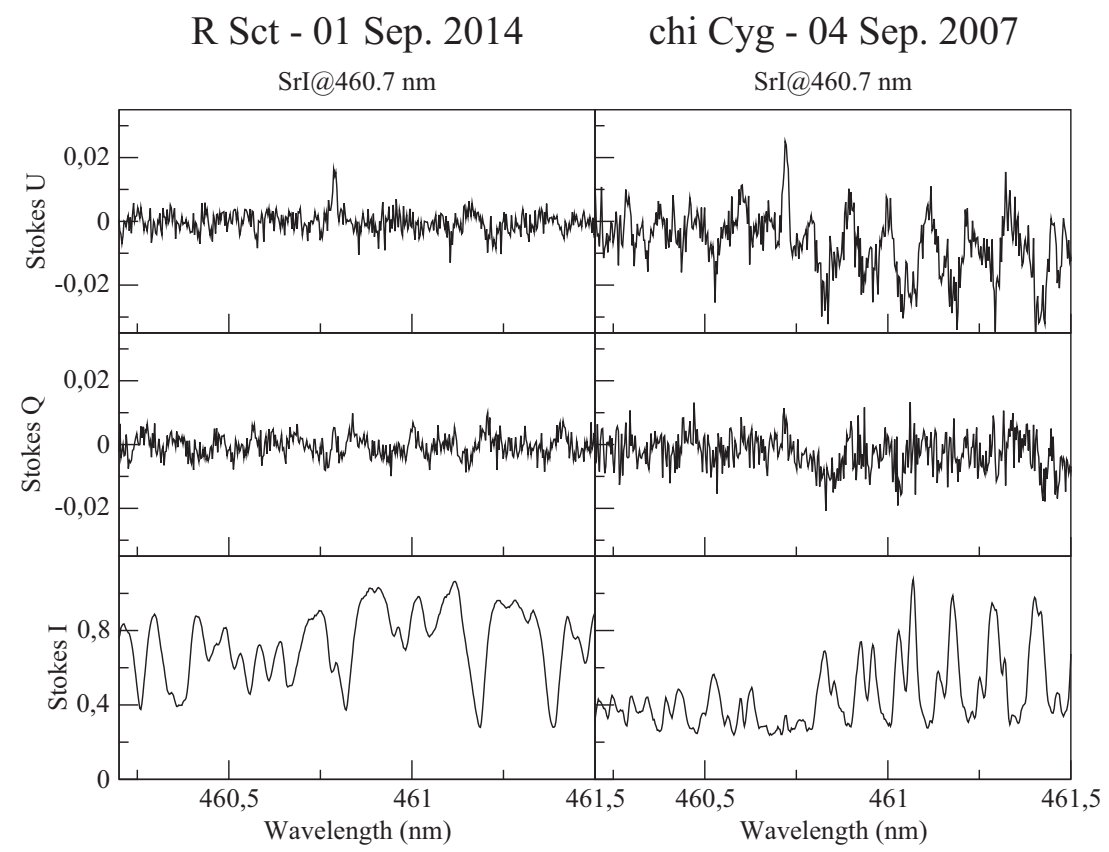

Figure 3. R Sct(left) and $\chi$ Cyg (right): From bottom to top panels, Stokes $I$, Stokes $Q$ and Stokes $U$ spectra in the region of the atomic line SrI@460.7 nm. Narval observations collected respectively on the 1st September 2014 (R Sct) and on the 7th September 2007 ( $\chi$ Cyg).

and illustrated in Fig.1 of Lèbre et al. (2014). For R Sct, we present in Figure 1 the LSD profiles (Stokes $I$, Stokes $V$ and the Null profile that does not contain any feature within the line profile when a Zeeman signature is present in the Stokes $V$ spectrum) for three observations collected with Narval along the summer 2014 when the star was just at (July 2014) and after (September 2014) a maximum light. A Zeeman signature is detected in the July date (as already reported in Sabin et al. 2015) and also confirmed in the early September date (with a significant detection statistics). However, this feature has almost disappeared in the mid-September date, when the shock wave is supposed to propagate in the highest layers of the stellar atmosphere. For these three dates, the longitudinal component of the magnetic field remains quite constant and leads to a surface field estimation of about $1 \mathrm{G}$. For R Sct, we also notice that the faint signals detected in the Stokes $V$ spectra can appear to be slightly shifted toward the blue part of the Stokes $I$ profiles (in the July observation) or toward the red part of the Stokes $I$ profiles (in the last observation of September), tracing the dynamical impact of the shock propagation on the photospheric layers, as it has been also reported for $\chi$ Cyg in Lèbre et al. (2014).

\section{Linear polarization within atomic lines}

When processing the linear polarization data with the LSD software we have also detected in both stars, $\chi \mathrm{Cyg}$ and $\mathrm{R}$ Sct, strong Stokes $U$ and Stokes $Q$ signatures associated to atomic lines. These detections appear to reflect a global trend affecting metallic lines in the same way as those LSD profiles are obtained from the combination of several thousands of atomic lines (supposed to be formed in the lower part of the stellar atmosphere). For $\chi$ Cyg, Lèbre et al. (2014) have reported such linear polarization signatures associated to metallic lines through Stokes $U \&$ Stokes $Q$ data collected (with 


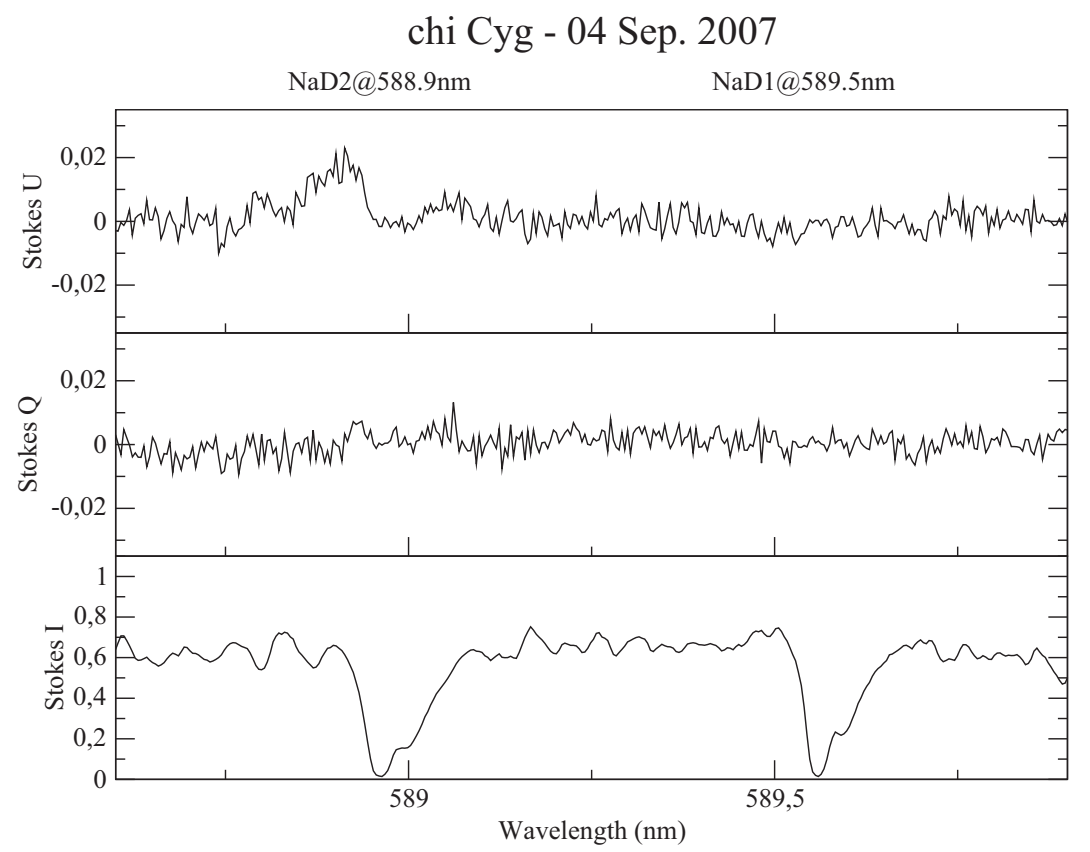

Figure 4. $\chi$ Cyg: From bottom to top panels, Stokes $I$, Stokes $Q$ and Stokes $U$ spectra in the region of the Sodium doublet lines (in the 4th September 2007 observation, at a max. light).

Narval) at the maximum light of 2007 (see their Fig. 2). Moreover, in the case of R Sct, we have established that those equivalent features were time variable along the pulsation phase. Figure 2 shows the LSD profiles (in Stokes $I$, Stokes $Q$ \& Stokes $U$ ) for the three observations of R Sct collected during the summer 2014. While the LSD statistics proposes a No Detection flag for the July 2014 observation, we notice an important variation in the shape and intensity of the Stokes $U \&$ Stokes $Q$ profiles in the observations collected in September 2014 ( resulting in Definite Detection flags in the LSD statistics).

In the Mira star $\chi \mathrm{Cyg}$, we have also detected linear polarization signatures associated to individual atomic lines. First, Fabas et al. (2011) have revealed for the prototypical Mira star, omi Cet, the time variable behavior of the linear polarization signatures associated to the Hydrogen Balmer lines emissions. These lines are indeed formed in the radiative wake of the shock wave periodically propagating throughout the stellar atmosphere, and hence their associated linear polarization signals present variable structures well connected to the presence and to the evolution of such an atmospheric shock wave. An interaction of the shock front and the convective pattern is suspected to result into a noticeable asymmetry at the photospheric level.

Surprisingly, for R Sct and for $\chi$ Cyg, we also report linear polarization structures associated to individual metallic lines, such as the SrI@460.7 nm or the NaD2@588.9 nm. From the solar case and the analyzis of the linearly polarized spectrum (the so-called Second Solar Spectrum), those lines are known to be easily polarizable in case of a global asymmetry at the photospheric level. Figure 3 illustrates the case of the linear polarization associated to the SrI@460.7 nm detected in the September 2014 observation of R Sct, and in the September 2007 observation of $\chi$ Cyg. Moreover, in this latter case, we have also detected polarization features associated to the Sodium NaD2 line, while no noticeable linear polarizable is detected within the NaD1 line (see Figure 4). This behavior is also well known from the solar case (and its origin is still debated). 
Considering the current explanations of the solar case, we suspect - in the cases of the Mira star, $\chi$ Cyg, and the RV Tauri star, R Sct - the same scenario to be at the origin of these features: a global asymmetry at the photospheric level resulting - after a scattering process - into a net linear polarization that we can detect in the Stokes $U$ and Stokes $Q$ data. Hence, we propose that the interaction of the shock front with giant convective cells present at the photospheric level would generate such a global asymmetry.

We therefore intend to monitor the cool and evolved pulsating targets, Mira and RV Tauri stars, along a large part of their pulsation cycle, so as to precise the occurence of the reported structures in both circular and linear polarization channels, and to study their connection with the atmospheric dynamics and the shock wave propagation. A Large Program with Narval@TBL is about to start, and it will allow the regular collect of full Stokes spectroplarimetric measurements on the brightest targets among Mira and RV Tauris stars, including $\chi$ Cyg and R Sct.

\section{Acknowledgement}

We acknowledge financial support from "Programme National de Physique Stellaire (PNPS) of CNRS/INSU, France."

\section{References}

Donati, J. F., Semel, M., Carter, B., \& Reed, D., Collier Cameron A., 1997, MNRAS 291, 658 Dorch, S. 2004 A $\& A 423,1101$

Fabas, N., Lèbre, A., \& Gillet, D. 2011, A\&A 535, A12

Herpin, F., Baudry, A., Thum, C., Morris, D., \& Wiesemeyer, H. 2006, A $\& A 450,667$

Lèbre, A., Aurière, M., Fabas, N., Gillet, D., Herpin, F., Konstantinova-Antova, R., \& Petit, P. 2014, A\&A 561, A85

Pascoli, G. \& Lahoche, L. 2008, PASP 129, 1267

Sabin, L., Wade, G., \& Lèbre, A. 2015, MNRAS 446, 1988

Thirumalai, A. \& Heyl, J. S. 2012, MNRAS 409, 1669

Van Winckel, H., Waelkens, C., Fernie, J. D., \& Waters, L. B. F.. M. 1999, A\&A 343, 202

Vlemmings, W. H. T. 2011, in: A. Zijlstra et al. (eds.), Asymmetric Planetary Nebulae 5 p. 91

Willson, L. A. 2000, ARAE\&A 38, 573 